Louisiana State University

LSU Digital Commons

Faculty Publications

Department of Mathematics

7-28-2016

\title{
Stabilization in a chemostat with sampled and delayed measurements
}

\author{
Frédéric Mazenc \\ Laboratoire des Signaux et Systèmes \\ Jérome Harmand \\ Laboratoire de Biotechnologie de l'Environnement (LBE) \\ Michael Malisoff \\ Louisiana State University
}

Follow this and additional works at: https://digitalcommons.Isu.edu/mathematics_pubs

\section{Recommended Citation}

Mazenc, F., Harmand, J., \& Malisoff, M. (2016). Stabilization in a chemostat with sampled and delayed measurements. Proceedings of the American Control Conference, 2016-July, 1857-1862. https://doi.org/ 10.1109/ACC.2016.7525189

This Conference Proceeding is brought to you for free and open access by the Department of Mathematics at LSU Digital Commons. It has been accepted for inclusion in Faculty Publications by an authorized administrator of LSU Digital Commons. For more information, please contact ir@lsu.edu. 


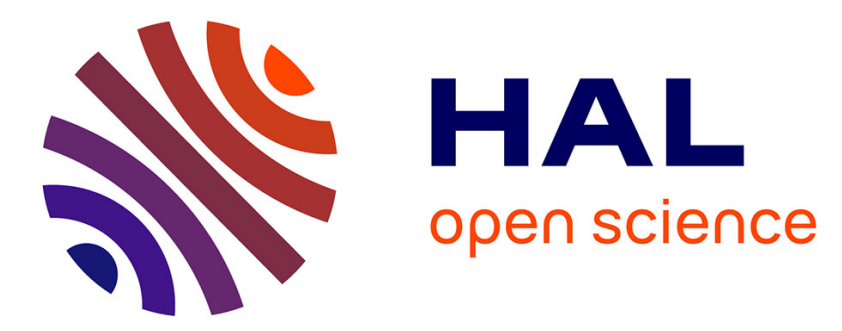

\title{
Stabilization in a chemostat with sampled and delayed measurements
}

\author{
Frédéric Mazenc, Jérome Harmand, Michael Malisoff
}

\section{To cite this version:}

Frédéric Mazenc, Jérome Harmand, Michael Malisoff. Stabilization in a chemostat with sampled and delayed measurements. 2016 American Control Conference (ACC), Jul 2016, Boston, United States. pp.1857 - 1862, 10.1109/ACC.2016.7525189 . hal-01389864

\section{HAL Id: hal-01389864 \\ https://hal.inria.fr/hal-01389864}

Submitted on 30 Oct 2016

HAL is a multi-disciplinary open access archive for the deposit and dissemination of scientific research documents, whether they are published or not. The documents may come from teaching and research institutions in France or abroad, or from public or private research centers.
L'archive ouverte pluridisciplinaire $\mathbf{H A L}$, est destinée au dépôt et à la diffusion de documents scientifiques de niveau recherche, publiés ou non, émanant des établissements d'enseignement et de recherche français ou étrangers, des laboratoires publics ou privés. 


\section{Stabilization in a Chemostat with Sampled and Delayed Measurements}

Frédéric Mazenc

\author{
Jérome Harmand
}

\author{
Michael Malisoff
}

\begin{abstract}
We study control problems for chemostat models with one species, one limiting substrate, and a constant substrate input concentration. We allow Haldane growth functions and other growth functions that are not necessarily monotone. The measurement is assumed to be the substrate concentration, which is piecewise constant with a constant delay. Under conditions on the size of the delay and on the largest sampling interval, we solve the problem of asymptotically stabilizing a componentwise positive equilibrium point with the dilution rate as control. We use a new type of Lyapunov approach.
\end{abstract}

Key Words: output-feedback, stabilization, delay, sampling

\section{INTRODUCTION}

The chemostat is a laboratory device used for the continuous culture of microorganisms. It was proposed simultaneously by Monod and Novick and Szilard in the 1950s; see [11], [12]. Today, it is often viewed in biotechnology, ecology, and microbiology as an ideal representation for modeling microorganisms or cells growth rates, wastewater treatment processes, or any natural environment such as a lake [4], [5], [7], [15]. The dynamics of the main variables evolving in the chemostat (which are basically the microorganisms and substrates concentrations) are usually based on mass-balance equations described by various mathematical models [1], [2], [16]. The problem of controlling such models is often difficult, notably because of the nonlinearity of the equations. Also, these systems like many other biological systems suffer from a lack of online sensors and actuators.

Even more crucial is the fact that online devices, such as those used for measuring substrate or biomass concentrations, deliver discrete variables with delays when they are available. In practice, the control laws are usually designed using continuous models and are discretized before being applied on the real system. Practitioners then rely on the robustness of control laws with respect to measurement delays in order for the control to realize its objectives. Measurement delays and their discrete nature are not the only disturbances the user has to deal with and it is expected that control performances could be improved if these characteristics are taken into account at the control design step. To

Mazenc is with EPI DISCO INRIA-Saclay, Laboratoire des Signaux et Systèmes (L2S, UMR CNRS 8506), CNRS, CentraleSupélec, Université Paris-Sud, 3 rue Joliot Curie, 91192, Gif-sur-Yvette, France. frederic.mazencel2s. centralesupelec.fr. Supported by l'Institut pour le Contrôle et la Décision de l'Idex Paris-Saclay (iCODE).

Harmand is with UR0050, LBE-INRA, Avenue des étangs, 11100 Narbonne and EPI INRIA MODEMIC, UMR MISTEA, Montpellier, France Jerome. Harmandesupagro. inra.fr.

Malisoff is with the Department of Mathematics, 303 Lockett Hall, Louisiana State University (LSU, Baton Rouge, LA 70803-4918, USA. malisoff@lsu.edu. Supported by NSF-ECCS Grants 1102348 and 1408295 and Roy P. Daniels Professorship \#3 in LSU College of Science. the best of the authors' knowledge, no rigorous theoretical study addresses this problem, except for [8]. The paper [14] assumes that the measurements are continuous.

These remarks motivated [8] and the present work, which complements [8]. In this paper, we consider the classical model of the chemostat described in [16] with one substrate and one species. The growth rate is assumed to be of Haldane type (i.e., having a limitation of the growth for low substrate concentrations and inhibition at high concentrations) and the input substrate concentration is assumed to be constant. The dilution rate is used as a control. Controlling this system is a challenging problem, for two main reasons. First, results such as [10] that establish global asymptotic stability under suitable bounds on the delay and sampling intervals are based on state feedbacks and certain strict Lyapunov function constructions. The work in this paper entails output feedbacks, and so is beyond the scope of [10].

Second, the model generally admits two equilibria when the dilution rate is constant. One is locally exponentially stable and the other is unstable. In [8], the problem of stabilizing points of the first type was addressed, while here we stabilize points of the second type, i.e., under inhibition at high substrate concentration as modeled in the Haldane function. For this latter case, we construct a stabilizing control law that only requires measurements of the substrate concentration, which are assumed to be piecewise constant and with constant delay. The control law ensures asymptotic convergence to the equilibrium point, when suitable bounds on the size of the delay and on the largest sampling interval are satisfied. This contrasts with [8], where no constraint of this type was imposed. The reason why this extra constraint is needed is that we stabilize points which can be exponentially unstable when a constant dilution rate is chosen.

In this work, we design a new feedback, and perform a stability analysis for the system in closed loop with the feedback. It is reminiscent of the theoretical contribution of [10]. However, the main result of [10] does not apply directly, so an ad hoc proof has to be proposed. Our proof is also very different from the one in [9], which is based on the assumptions that the growth rates are given by a known analytic expression of Haldane or Monod type and that measurements for the species levels are available in the control design, making it possible to design Lyapunov functionals.

\section{THE MODEL}

We consider the following model of chemostat:

$$
\left\{\begin{array}{l}
\dot{s}(t)=D\left[s_{i n}-s(t)\right]-\mu(s(t)) x(t) \\
\dot{x}(t)=[\mu(s(t))-D] x(t)
\end{array}\right.
$$


where $s_{i n}>0$ is a constant, and where the states $x$ and $s$ are positive and real valued, and where $D$ is a positive valued control. In what follows, $C^{1}$ means continuously differentiable.

Assumption 1: The function $\mu$ is of class $C^{1}$ and $\mu(0)=$ 0 . There is a constant $s_{M}>0$ such that $\mu^{\prime}(s)>0$ for all $s \in\left[0, s_{M}\right)$ and $\mu^{\prime}(s) \leq 0$ for all $s \in\left(s_{M},+\infty\right)$. Finally, $\mu(s)>0$ for all $s>0$.

Assumption 1 is satisfied by all functions of the form

$$
\mu(s)=\frac{k_{1} s}{1+k_{2} s+k_{3} s^{2}},
$$

where $k_{i}>0$ for $i=1$ to 3 are arbitrary constants. Functions of the form (2) are called Haldane functions. Moreover, we can prove:

Lemma 1: If Assumption 1 holds, then we can find a $C^{1}$ class $\mathcal{K}_{\infty}$ function $\mu_{1}$ and a nondecreasing $C^{1}$ function $\gamma$ : $\mathbb{R} \rightarrow[0,+\infty)$ such that $\gamma(m)=0$ for all $m \leq 0$ and such that

$$
\mu(s)=\frac{\mu_{1}(s)}{1+\gamma(s)}
$$

holds for all $s \geq 0$.

For the proof of Lemma 1, see Appendix VI.

Remark 1: When $s_{i n}>s_{M}$ and $D$ is constant such that $D \in\left(\mu\left(s_{i n}\right), \mu\left(s_{M}\right)\right)$, then one can easily check that the system (1) admits a locally stable equilibrium $\left(s_{i n}, 0\right)$ and a locally unstable positive equilibrium point of the form $\left(s_{*}, s_{i n}-s_{*}\right)$. Here $s_{*}>0$ is such that $D=\mu\left(s_{*}\right)$ and $s_{*} \in\left(s_{M}, s_{i n}\right)$. In [8], we solved the problem of globally stabilizing an equilibrium point that can be locally exponentially stabilized through a constant dilution rate.

To make our sampling control objective precise, fix any two constants $\epsilon_{1}>0$ and $\epsilon_{2}>0$ such that $\epsilon_{2}>\epsilon_{1}$, and let $t_{i}$ be a sequence of real numbers such that $t_{0}=0$ and such that

$$
0<\epsilon_{1} \leq t_{i+1}-t_{i} \leq \epsilon_{2}
$$

for all $i \in \mathbb{N}$, where $\mathbb{N}=\{1,2, \ldots\}$. We let $\tau_{f} \geq 0$ be a constant, and we define the function $\tau$ by

$$
\begin{aligned}
& \tau(t)= \\
& \begin{cases}\tau_{f}, & t \in\left[0, \tau_{f}\right) \\
\tau_{f}+t-t_{j}, & t \in\left[t_{j}+\tau_{f}, t_{j+1}+\tau_{f}\right) \text { and } j \geq 0\end{cases}
\end{aligned}
$$

which is reminiscent of the representation of sampling used for instance in [3]. From this definition, it follows that when $t \in\left[t_{j}+\tau_{f}, t_{j+1}+\tau_{f}\right)$, then $t-\tau(t)=t-\left(\tau_{f}+t-t_{j}\right)=$ $t_{j}-\tau_{f}$. Thus, the function $t-\tau(t)$ is piecewise constant. For instance, when $\tau_{f}=0$ and $t_{j}=j$ for all $j \geq 0$, then $t-\tau(t)=j$ for all $t \in[j, j+1)$ and $j \geq 0$.

Notice for later use that for all $t \geq 0$, we have

$$
0 \leq \tau(t) \leq \tau_{M}
$$

where

$$
\tau_{M}=\tau_{f}+\epsilon_{2}
$$

We assume that the only measurement available is $s(t-\tau(t))$. Our control objective is the asymptotic stabilization of the point $E_{*}=\left(s_{*}, s_{i n}-s_{*}\right)$ for any constant $s_{*} \in\left(0, s_{i n}\right)$, with a nonnegative feedback of the form $D(s(t-\tau(t)))$. Notice that $E_{*}$ is positive and is an equilibrium point of (1) if and only if $D\left(s_{*}\right)=\mu\left(s_{*}\right)$.

\section{MAIN RESUlT}

Under our Assumption 1, we fix any functions $\mu_{1}$ and $\gamma$ that satisfy the requirements from Lemma 1 and any constants $s_{i n}>0$ and $s_{*} \in\left(0, s_{i n}\right)$. We use the constants

$$
\begin{gathered}
\mu_{a}=\mu_{1}\left(s_{*}\right) s_{i n}, \\
\varpi_{s}=\inf _{s \in\left[0, s_{i n}\right]} \mu_{1}^{\prime}(s), \varpi_{l}=\sup _{s \in\left[0, s_{i n}\right]} \mu_{1}^{\prime}(s), \\
\rho_{l}=\sup _{s \in\left[0, s_{i n}\right]} \gamma^{\prime}(s),
\end{gathered}
$$

and

$$
\rho_{m}=\frac{\rho_{l}^{2}}{2 \varpi_{s}} \sup _{l \in\left[0, s_{i n}\right]} \frac{\mu_{1}\left(l+1.1 \mu_{a} \tau_{M}\right)^{2}}{1+\gamma(l)}
$$

where $\tau_{M}$ is from (7). The properties of $\mu_{1}$ and $\gamma$ ensure that they are well-defined and positive. Finally, we assume:

Assumption 2: The constant $\tau_{M}$ from (7) is such that

$$
\begin{gathered}
\frac{\mu_{1}\left(s_{i n}\right)}{1+\gamma\left(s_{i n}\right)}-\frac{\mu_{1}\left(s_{*}\right)}{1+\gamma\left(s_{i n}-\mu_{a} \tau_{M}\right)}>0, \\
\tau_{M}<\frac{1}{2 \sqrt{2 \rho_{m} \varpi_{l}} s_{i n}}, \text { and } \tau_{M} \leq \frac{1}{2 \rho_{l} s_{i n} \mu\left(s_{M}\right)}
\end{gathered}
$$

are all satisfied.

Since $s_{*}<s_{\text {in }}$ and $\mu_{1}$ is increasing, it is usually easy to determine a constant $\bar{\tau}>0$ such that (11) is satisfied for all $\tau_{M} \in(0, \bar{\tau})$. See the example below where we check our assumptions. Our main result is then:

Theorem 1: If Assumptions 1-2 hold, then for each constant $s_{*} \in\left(0, s_{i n}\right)$, all solutions of (1) in closed loop with

$$
D(t)=\frac{\mu_{1}\left(s_{*}\right)}{1+\gamma(s(t-\tau(t)))}
$$

asymptotically converge to $\left(s_{*}, s_{i n}-s_{*}\right)$.

\section{PRoOF OF TheOREM 1}

Fix any continuous initial function $\left(\phi_{s}, \phi_{x}\right):\left[-\tau_{M}, 0\right] \rightarrow$ $(0, \infty)^{2}$ for the corresponding closed loop system

$$
\left\{\begin{array}{l}
\dot{s}(t)=\frac{\mu_{1}\left(s_{*}\right)}{1+\gamma(s(t-\tau(t)))}\left[s_{i n}-s(t)\right]-\mu(s(t)) x(t) \\
\dot{x}(t)=\left[\mu(s(t))-\frac{\mu_{1}\left(s_{*}\right)}{1+\gamma(s(t-\tau(t)))}\right] x(t) .
\end{array}\right.
$$

We must prove that this initial condition generates a positive valued solution $(x(t), s(t))$ of the system (14) that defined over $[0,+\infty)$, positive and such that

$$
\lim _{t \rightarrow+\infty}(s(t), x(t))=\left(s_{*}, s_{i n}-s_{*}\right) .
$$

First Step. One can easily prove that the solution $(s(t), x(t))$ is defined over $\left[-\tau_{M},+\infty\right)$ and positive valued, since $D(t) s_{\text {in }}>0$ would give $\dot{s}(t)>0$ at any time $t$ when $s(t)=0$. We next prove that the solution is bounded. Set

$$
z(t)=s_{\text {in }}-s(t)-x(t) .
$$

An elementary calculation gives

$$
\dot{z}(t)=-\frac{\mu_{1}\left(s_{*}\right)}{1+\gamma(s(t-\tau(t)))} z(t) .
$$


Here and in the sequel, all equalities and inequalities should be understood to hold for all $t \geq 0$ unless otherwise noted. Consequently, $|z(t)| \leq|z(0)|$ for all $t \geq 0$. It follows that for all $t \geq 0$, we have

$$
\begin{aligned}
|(x(t), s(t))| & \leq s(t)+x(t)=-z(t)+s_{i n} \\
& \leq|z(0)|+s_{i n} \leq c_{a}
\end{aligned}
$$

where $c_{a}=s_{\text {in }}+\left|s_{\text {in }}-s(0)-x(0)\right|$. Also, (17)-(18) give

$$
|z(t)| \leq\left|z\left(\tau_{M}\right)\right| e^{\frac{\mu_{1}\left(s_{*}\right)}{1+\gamma\left(c_{a}\right)}\left(\tau_{M}-t\right)}
$$

for all $t \geq \tau_{M}$.

Second step. We prove by contradiction that there is a $t_{a}>2 \tau_{M}$ such that $s\left(t_{a}\right) \in\left(0, s_{i n}\right)$. Assume that for all $t>2 \tau_{M}$, we have $s(t) \geq s_{i n}$. Then for all $t>2 \tau_{M}$, we get

$$
\begin{aligned}
& -\mu(s(t)) x(t)<0 \text { and } \\
& \frac{\mu_{1}\left(s_{*}\right)}{1+\gamma(s(t-\tau(t)))}\left[s_{i n}-s(t)\right] \leq 0 .
\end{aligned}
$$

Hence, $\dot{s}(t)<0$ for all $t>2 \tau_{M}$. Since $s(t)$ is lower bounded by $s_{i n}$, it follows that $s(t)$ converges to some limit $s_{l}$ such that $s_{l} \geq s_{i n}$. Since $s(t)=s_{i n}-x(t)-z(t)<s_{\text {in }}-z(t)$ and $z(t)$ converges to zero, we get $s_{l}=s_{i n}$. Therefore,

$$
\begin{aligned}
& \lim _{t \rightarrow+\infty} \mu(s(t))=\frac{\mu_{1}\left(s_{i n}\right)}{1+\gamma\left(s_{i n}\right)} \text { and } \\
& \lim _{t \rightarrow+\infty} \frac{\mu_{1}\left(s_{*}\right)}{1+\gamma(s(t-\tau(t)))}=\frac{\mu_{1}\left(s_{*}\right)}{1+\gamma\left(s_{i n}\right)} .
\end{aligned}
$$

Since $\mu_{1}$ is increasing and $s_{i n}>s_{*}$, we deduce from the two equalities in (21) that there is $t_{b}>t_{a}$ such that for all $t \geq t_{b}, \mu(s(t))-\frac{\mu_{1}\left(s_{*}\right)}{1+\gamma(s(t-\tau(t)))} \geq c_{b}$, where

$$
c_{b}=\frac{\mu_{1}\left(\frac{s_{i n}+s_{*}}{2}\right)}{1+\gamma\left(s_{i n}\right)}-\frac{\mu_{1}\left(s_{*}\right)}{1+\gamma\left(s_{\text {in }}\right)}>0 .
$$

It follows from (14) that $\dot{x}(t) \geq c_{b} x(t)$ holds for all $t \geq t_{b}$, so $\lim _{t \rightarrow+\infty} x(t)=+\infty$. This contradicts the fact that $x(t)$ is bounded. Since $D$ is positive valued, we conclude that there is $t_{a}>2 \tau_{M}$ such that $s(t) \in\left(0, s_{i n}\right)$ for all $t \geq t_{a}$, since the structure of the dynamics ensure that we would have $\dot{s}(t)<0$ at any time $t$ when $s(t) \geq s_{\text {in }}$.

Third step. We prove that there are constants $t_{c} \geq t_{a}$ and $s_{\triangle} \in\left(0, s_{\text {in }}\right)$ such that for all $t \geq t_{c}$, we have $s(t)<s_{\triangle}$.

With $z$ defined in (16), we can use the fact that $s(t)=$ $s_{\text {in }}-z(t)-x(t)$ to get

$$
\begin{aligned}
\dot{x}(t)= & {\left[\frac{\mu_{1}\left(s_{i n}-x(t)-z(t)\right)}{1+\gamma\left(s_{i n}-x(t)-z(t)\right)}\right.} \\
& \left.-\frac{\mu_{1}\left(s_{*}\right)}{1+\gamma\left(s_{i n}-x(t)-z(t)+s(t-\tau(t)-s(t))\right)}\right] x(t) .
\end{aligned}
$$

Also, for all $t \geq 2 \tau_{M}$, we get

$$
s(t-\tau(t))-s(t)=-\int_{t-\tau(t)}^{t} \dot{s}(m) d m
$$

and

$$
\begin{aligned}
& \int_{t-\tau(t)}^{t} \dot{s}(m) d m \\
& =\int_{t-\tau(t)}^{t}\left(\frac{\mu_{1}\left(s_{*}\right)}{1+\gamma(s(t-\tau(t)))}-\frac{\mu_{1}(s(m))}{1+\gamma(s(m))}\right)\left[s_{i n}-s(m)\right] d m \\
& +\int_{t-\tau(t)}^{t} \frac{\mu_{1}(s(m))}{1+\gamma(s(m))} z(m) d m .
\end{aligned}
$$

Since $s_{\text {in }}-s(t) \geq 0$ holds for all $t \geq t_{a}$, it follows that for all $t \geq t_{a}+2 \tau_{M}$ we get

$$
\begin{aligned}
& \int_{t-\tau(t)}^{t} \dot{s}(m) d m \leq \\
& \mu_{a} \tau_{M}+\mu_{1}\left(s_{i n}\right) \int_{t-\tau(t)}^{t}|z(m)| d m,
\end{aligned}
$$

where $\mu_{a}$ was defined in (8) and $\tau_{M}$ is from (7).

Let $q$ denote the left side of (11) from Assumption 2. Since $\gamma$ is nondecreasing, we deduce from (23), (24) and (25) and the fact that $x(t) \leq c_{a}$ for all $t \geq 0$ that

$$
\dot{x}(t) \geq\left[q+\kappa(x(t))+\lambda\left(x_{t}, z_{t}\right)\right] x(t)
$$

holds for all $t \geq t_{a}+2 \tau_{M}$, where

$$
\begin{aligned}
& \lambda\left(x(t), z_{t}\right)=\frac{\mu_{1}\left(s_{i n}-x(t)-z(t)\right)}{1+\gamma\left(s_{i n}-x(t)-z(t)\right)}-\frac{\mu_{1}\left(s_{i n}-x(t)\right)}{1+\gamma\left(s_{i n}-x(t)\right)} \\
& +\frac{\mu_{1}\left(s_{*}\right)}{1+\gamma\left(s_{i n}-x(t)-\mu_{a} \tau_{M}\right)} \\
& -\frac{\mu_{1}\left(s_{*}\right)}{1+\gamma\left(s_{i n}-x(t)-\mu_{a} \tau_{M}-z(t)-\mu_{1}\left(s_{i n}\right) \int_{t-\tau(t)}^{t}|z(m)| d m\right)}
\end{aligned}
$$

and

$$
\begin{aligned}
\kappa(x)= & \frac{\mu_{1}\left(s_{i n}-x\right)}{1+\gamma\left(s_{i n}-x\right)}-\frac{\mu_{1}\left(s_{i n}\right)}{1+\gamma\left(s_{i n}\right)} \\
& +\frac{\mu_{1}\left(s_{*}\right)}{1+\gamma\left(s_{i n}-\mu_{a} \tau_{M}\right)}-\frac{\mu_{1}\left(s_{*}\right)}{1+\gamma\left(s_{i n}-x-\mu_{a} \tau_{M}\right)} .
\end{aligned}
$$

Since $\lim _{t \rightarrow+\infty} z(t)=0$, we get $\lim _{t \rightarrow \infty} \lambda\left(x(t), z_{t}\right)=0$ so we deduce from Assumption 2 that there is a $t_{c}>2 \tau_{M}$ such that for all $t \geq t_{c}$, we have

$$
\dot{x}(t) \geq\left[\frac{q}{2}+\kappa(x(t))\right] x(t) .
$$

Since the function $\kappa$ is continuous and $\kappa(0)=0$, there exists $x_{p} \in\left(0, s_{i n}\right)$ such that for all $x \in\left[0, x_{p}\right]$, we have $\kappa(x) \geq$ $-\frac{q}{4}$. One can then prove that there is a $t_{e} \geq t_{d}$ such that for all $t \geq t_{e}$, we have

$$
x(t) \geq x_{p} .
$$

This follows because $\dot{x}(t) \geq q x(t) / 4$ at all times $t$ when $x(t) \in\left(0, x_{p}\right]$. Also, (19) implies that there is a $t_{f} \geq t_{e}$ such that for all $t \geq t_{f}$, the inequality

$$
|z(t)| \leq \frac{x_{p}}{4}
$$

holds. By using the definition of $z,(31)$ and (30), we deduce that for all $t \geq t_{f}$, we have

$$
s(t) \leq-x(t)+\frac{x_{p}}{4}+s_{i n} \leq-x_{p}+\frac{x_{p}}{4}+s_{i n}=s_{\triangle},
$$

where $s_{\triangle}=s_{i n}-\frac{3 x_{p}}{4}$. Since $x_{p} \in\left(0, s_{i n}\right)$, it follows that $s \triangle \in\left(0, s_{\text {in }}\right)$.

Fourth step. We build a functional $\mathcal{U}_{1}$ that is reminiscent of barrier Lyapunov functions that are used in adaptive control and parameter identification. Later, we add double integral term to $\mathcal{U}_{1}$ to prove our stability property. Using $z$ defined in (16) gives the following for all $t \geq t_{M}$ :

$$
\begin{aligned}
\dot{s}(t)= & {\left[\frac{\mu_{1}\left(s_{*}\right)}{1+\gamma(s(t-\tau(t)))}-\frac{\mu_{1}(s(t))}{1+\gamma(s(t))}\right]\left[s_{i n}-s(t)\right] } \\
& +\mu(s(t)) z(t) .
\end{aligned}
$$

Since $\gamma(s(t))-\gamma(s(t-\tau))=\int_{t-\tau(t)}^{t} \gamma^{\prime}(s(m)) \dot{s}(m) d m$ holds for all $t \geq 2 \tau_{M}$, we can use (33) to get

$$
\begin{aligned}
& \dot{s}(t)=\mu(s(t)) z(t)+ \\
& \frac{\left[\mu_{1}\left(s_{*}\right)-\mu_{1}(s(t))\right][1+\gamma(s(t))]+\mu_{1}(s(t)) \mathcal{I}(t)}{[1+\gamma(s(t-\tau(t)))][1+\gamma(s(t))]}\left[s_{i n}-s(t)\right]
\end{aligned}
$$


for all $t \geq 2 \tau_{M}$, where $\mathcal{I}(t)=\int_{t-\tau(t)}^{t} \gamma^{\prime}(s(m)) \dot{s}(m) d m$.

We use

$$
\mathcal{U}_{1}(s)=\int_{0}^{s-s_{*}} \frac{m}{s_{i n}-s_{*}-m} d m,
$$

which is of class $C^{1}$ over $\left[0, s_{i n}\right)$ and nonnegative valued. It follows from (34) that its derivative along all trajectories of the closed loop system for all $t \geq t_{a}+2 \tau_{M}$ satisfies

$$
\begin{aligned}
& \dot{\mathcal{U}}_{1}(t)=\frac{s(t)-s_{*}}{s_{i n}-s(t)} \mu(s(t)) z(t)+ \\
& \frac{\left[\mu_{1}\left(s_{*}\right)-\mu_{1}(s(t))\right][1+\gamma(s(t))]+\mu_{1}(s(t)) \mathcal{I}(t)}{[1+\gamma(s(t-\tau(t)))][1+\gamma(s(t))]}\left[s(t)-s_{*}\right] .
\end{aligned}
$$

From the third step, we deduce that when $t \geq t_{c}+2 \tau_{M}$, the inequality

$$
\begin{aligned}
& \dot{\mathcal{U}}_{1}(t) \leq \\
& \frac{\left(s(t)-s_{*}\right)\left(\mu_{1}\left(s_{*}\right)-\mu_{1}(s(t))\right)}{1+\gamma(s(t-\tau(t)))}+\frac{\left|s(t)-s_{*}\right| \mu\left(s_{i n}\right)}{s_{i n}-s_{\triangle}}|z(t)| \\
& +\left|s(t)-s_{*}\right| \frac{\mu_{1}(s(t)) \int_{t-\tau(t)}^{t} \gamma^{\prime}(s(m))|\dot{s}(m)| d m}{1+\gamma(s(t-\tau(t)))}
\end{aligned}
$$

is satisfied. From the definition $\rho_{l}$ in (9) and the fact that $s(t)<s_{\delta}$ holds for all $t \geq t_{c}+2 \tau_{M}$, we deduce that

$$
\begin{aligned}
\dot{\mathcal{U}}_{1}(t) \leq & \frac{\left(s(t)-s_{*}\right)\left(\mu_{1}\left(s_{*}\right)-\mu_{1}(s(t))\right)}{1+\gamma(s(t-\tau(t)))} \\
& +\rho_{l}\left|s(t)-s_{*}\right| \frac{\mu_{1}(s(t)) \int_{t-\tau(t)}^{t}|\dot{s}(m)| d m}{1+\gamma(s(t-\tau(t)))} \\
& +\frac{\left|s(t)-s_{*}\right| \mu\left(s_{i n}\right)}{s_{i n}-s_{\triangle}}|z(t)| \\
\leq & \frac{\left(s(t)-s_{*}\right)\left(\mu_{1}\left(s_{*}\right)-\mu_{1}(s(t))\right)}{2[1+\gamma(s(t-\tau(t)))]} \\
& -\varpi_{s} \frac{\left(s(t)-s_{*}\right)^{2}}{2[1+\gamma(s(t-\tau(t)))]} \\
& +\rho_{l}\left|s(t)-s_{*}\right| \frac{\mu_{1}\left(s(t-\tau(t))+2.2 \mu_{a} \tau_{M}\right)}{1+\gamma(s(t-\tau(t)))} \\
& \times \int_{t-\tau(t)}^{t}|\dot{s}(m)| d m \\
& +\frac{\left|s(t)-s_{*}\right| \mu\left(s_{i n}\right)}{s_{i n}-s_{\triangle}}|z(t)|
\end{aligned}
$$

when $t$ is large enough. The last inequality in (38) is a consequence of the definition of $\varpi_{s}$ in (9), which implies that $\left(\mu_{1}\left(s_{*}\right)-\mu(s(t))\left(s_{*}-s(t)\right) \geq \varpi_{s}\left|s_{*}-s(t)\right|^{2}\right.$ when we use the Mean Value Theorem, combined with (17) and (25).

Consequently,

$$
\begin{aligned}
\dot{\mathcal{U}}_{1}(t) \leq & \frac{\left(s(t)-s_{*}\right)\left(\mu_{1}\left(s_{*}\right)-\mu_{1}(s(t))\right)}{2[1+\gamma(s(t-\tau(t)))]} \\
& +\frac{\rho_{l}^{2}}{2 \varpi_{s}} \frac{\mu_{1}\left(s(t-\tau(t))+1.1 \mu_{a} \tau_{M}\right)^{2}}{1+\gamma(s(t-\tau(t)))} \tau_{M} \\
& \times \int_{t-\tau(t)}^{t}|\dot{s}(m)|^{2} d m \\
& +\frac{\left|s(t)-s_{*}\right| \mu\left(s_{i n}\right)}{s_{i n}-s_{\triangle}}|z(t)| \\
\leq & \frac{\left(s(t)-s_{*}\right)\left(\mu_{1}\left(s_{*}\right)-\mu_{1}(s(t))\right)}{2[1+\gamma(s(t-\tau(t)))]} \\
& +\rho_{m} \tau_{M} \int_{t-\tau(t)}^{t}|\dot{s}(m)|^{2} d m \\
& +\frac{\left|s(t)-s_{*}\right| \mu\left(s_{i n}\right)}{s_{i n}-s_{\triangle}}|z(t)|
\end{aligned}
$$

where $\rho_{m}$ is defined in (10), where we used the triangle and Jensen inequalities and the third step of the proof.

Fifth step. We next transform our function $\mathcal{U}_{1}$ to obtain a new function $\mathcal{U}_{2}$ that is reminiscent of LyapunovKrasovskii functionals, but which does not satisfy the classical Lyapunov-Krasovskii conditions. However, we will use it to derive stability properties. We prove that its derivative is nonpositive when $t$ is above a certain constant. We introduce the functional $\mathcal{U}_{2}$, whose expression along the trajectories is

$$
\mathcal{U}_{2}\left(s_{t}\right)=\mathcal{U}_{1}(s(t))+2 \rho_{m} \tau_{M} \int_{t-\tau_{M}}^{t} \int_{\ell}^{t} \dot{s}(m)^{2} d m d \ell \text {. }
$$

Then elementary calculations give, for all $t \geq t_{c}$,

$$
\begin{aligned}
\dot{\mathcal{U}}_{2}(t) \leq & \frac{\left(s(t)-s_{*}\right)\left(\mu_{1}\left(s_{*}\right)-\mu_{1}(s(t))\right)}{2[1+\gamma(s(t-\tau(t)))]} \\
& -\rho_{m} \tau_{M} \int_{t-\tau(t)}^{t}|\dot{s}(m)|^{2} d m \\
& +2 \rho_{m} \tau_{M}^{2} \dot{s}(t)^{2}+\frac{\left|s(t)-s_{*}\right| \mu\left(s_{i n}\right)}{s_{i n}-s_{\triangle}}|z(t)|
\end{aligned}
$$

From (34), and from the general relation $(a+b)^{2} \leq 2 a^{2}+2 b^{2}$ for suitable values $a \geq 0$ and $b \geq 0$, we can find a constant $c_{d}>0$ such that

$$
\begin{aligned}
& \dot{\mathcal{U}}_{2}(t) \leq \frac{\left(s(t)-s_{*}\right)\left(\mu_{1}\left(s_{*}\right)-\mu_{1}(s(t))\right)}{2[1+\gamma(s(t-\tau(t)))]} \\
& -\rho_{m} \tau_{M} \int_{t-\tau(t)}^{t}|\dot{s}(m)|^{2} d m \\
& +\left[\frac{\left[\mu_{1}\left(s_{*}\right)-\mu_{1}(s(t))\right][1+\gamma(s(t))]+\mu_{1}(s(t)) \rho_{l} \int_{t-\tau(t)}^{t}|\dot{s}(m)| d m}{[1+\gamma(s(t-\tau(t)))][1+\gamma(s(t))]}\right]^{2} \\
& \quad \times 2 \rho_{m} \tau_{M}^{2}\left[s_{i n}-s(t)\right]^{2}+c_{d}|z(t)| .
\end{aligned}
$$

Using our definition of $\varpi_{l}$ in (9), it follows that

$$
\begin{aligned}
& \dot{\mathcal{U}}_{2}(t) \leq \\
& \frac{\left(s(t)-s_{*}\right)\left(\mu_{1}\left(s_{*}\right)-\mu_{1}(s(t))\right)}{2[1+\gamma(s(t-\tau(t)))]}-\rho_{m} \tau_{M} \int_{t-\tau(t)}^{t}|\dot{s}(m)|^{2} d m \\
& +4 \rho_{m} \tau_{M}^{2}\left[\frac{\left(\mu_{1}\left(s_{*}\right)-\mu_{1}(s(t))\right)^{2}}{[1+\gamma(s(t-\tau(t)))]^{2}}\right. \\
& \left.+\frac{\mu_{1}(s(t))^{2} \rho_{l}^{2} \tau_{M} \int_{t-\tau(t)}^{t}|\dot{s}(m)|^{2} d m}{[1+\gamma(s(t-\tau(t)))]^{2}[1+\gamma(s(t))]^{2}}\right]\left[s_{i n}-s(t)\right]^{2}+c_{d}|z(t)| \\
& \leq \frac{\left(s(t)-s_{*}\right)\left(\mu_{1}\left(s_{*}\right)-\mu_{1}(s(t))\right)}{2[1+\gamma(s(t-\tau(t)))]} \\
& +4 \rho_{m} \tau_{M}^{2} \varpi_{l} \frac{\left(s_{*}-s(t)\right)\left(\mu_{1}\left(s_{*}\right)-\mu_{1}(s(t))\right)}{[1+\gamma(s(t-\tau(t)))]^{2}}\left[s_{i n}-s(t)\right]^{2} \\
& +\left(4 \rho_{m} \tau_{M}^{2} \frac{\mu_{1}(s(t))^{2} \rho_{l}^{2}}{[1+\gamma(s(t-\tau(t)))]^{2}[1+\gamma(s(t))]^{2}}\left[s_{i n}-s(t)\right]^{2}-\rho_{m}\right) \\
& \quad \times \tau_{M} \int_{t-\tau(t)}^{t} \dot{s}(m)^{2} d m+c_{d}|z(t)| .
\end{aligned}
$$

This gives

$$
\begin{aligned}
\dot{\mathcal{U}}_{2}(t) \leq & {\left[-1+8 \rho_{m} \tau_{M}^{2} \varpi_{l} \frac{\left[s_{i n}-s(t)\right]^{2}}{[1+\gamma(s(t-\tau(t)))]}\right] } \\
& \times \frac{\left(s_{*}-s(t)\right)\left(\mu_{1}\left(s_{*}\right)-\mu_{1}(s(t))\right)}{2[1+\gamma(s(t-\tau(t)))]} \\
+ & \left(4 \tau_{M}^{2} \rho_{l}^{2} \frac{\mu(s(t))^{2}}{[1+\gamma(s(t-\tau(t)))]^{2}}\left[s_{i n}-s(t)\right]^{2}-1\right) \\
& \times \rho_{m} \tau_{M} \int_{t-\tau(t)}^{t} \dot{s}(m)^{2} d m+c_{d}|z(t)| \\
\leq & \left(8 \rho_{m} \varpi_{l} s_{i n}^{2} \tau_{M}^{2}-1\right) \frac{\left(s_{*}-s(t)\right)\left(\mu_{1}\left(s_{*}\right)-\mu_{1}(s(t))\right)}{2[1+\gamma(s(t-\tau(t)))]} \\
+ & \left(4 \rho_{l}^{2} \mu(s(t))^{2} s_{i n}^{2} \tau_{M}^{2}-1\right) \\
& \times \rho_{m} \tau_{M} \int_{t-\tau(t)}^{t} \dot{s}(m)^{2} d m+c_{d}|z(t)|
\end{aligned}
$$

We therefore conclude from our bounds (12) on $\tau_{M}$ from Assumption 2 that that there is a constant $c_{e}>0$ such that for all $t \geq t_{c}$, we have

$$
\dot{\mathcal{U}}_{2}(t) \leq-c_{e}\left(s(t)-s_{*}\right)^{2}+c_{d}|z(t)|
$$

Last step. Integrating (43) over $\left[t_{c}, t\right]$ with $t \geq t_{c}$ gives

$$
\begin{aligned}
\mathcal{U}_{2}(t)-\mathcal{U}_{2}\left(t_{c}\right) \leq & -c_{e} \int_{t_{c}}^{t}\left(s(m)-s_{*}\right)^{2} d m \\
& +c_{d} \int_{t_{c}}^{t}|z(m)| d m .
\end{aligned}
$$

Since $\mathcal{U}_{2}$ is nonnegative valued, it follows that $c_{e} \int_{t_{c}}^{t}\left(s(m)-s_{*}\right)^{2} d m \leq \mathcal{U}_{2}\left(t_{c}\right)+c_{d} \int_{t_{h}}^{t}|z(m)| d m$. 
Since the function $(s(t), x(t))$ is bounded, it follows that $\dot{s}(t)$ is bounded. Also, $z(m)$ is integrable, since (19) ensures that $|z(m)|$ exponentially decays to 0 . We deduce that $s(t)$ is uniformly continuous. It follows from Barbalat's lemma and the inequality (45) that $\lim _{t \rightarrow+\infty}\left(s(t)-s_{*}\right)=0$. This implies that $\lim _{t \rightarrow+\infty} s(t)=s_{*}$ and $\lim _{t \rightarrow+\infty} x(t)=s_{\text {in }}-$ $s_{*}$. This concludes the proof.

\section{EXAMPLE}

We use the growth rate and constant

$$
\mu(s)=\frac{0.5 s}{1+s^{2}} \text { and } s_{i n}=1 .
$$

Since

$$
\mu^{\prime}(s)=0.5 \frac{1-s^{2}}{\left(1+s^{2}\right)^{2}},
$$

Assumption 1 holds with $s_{M}=1$. Also, the requirements of Lemma 1 are met using $\mu_{1}(s)=0.5 s$ and $\gamma(s)=s^{2}$.

We take $s_{*}=0.25$. Then the constants from Section III above are $\varpi_{s}=\varpi_{l}=0.5, \rho_{l}=2, \mu_{a}=0.2$, and

$$
\rho_{m}=\max _{\ell \in[0,1]} \frac{\left(\ell+0.1375 \tau_{M}\right)^{2}}{1+\ell^{2}} .
$$

If $\tau_{M}<1$, then the maximum in (48) occurs at $\ell=1$, so $\rho_{m} \leq 0.5(1+0.1375)^{2}=0.6469$. Also our condition (11) from Assumption 2 reads

$$
\frac{0.5}{2}-\frac{0.5(0.25)}{1+\left(1-0.2 \tau_{M}\right)^{2}}>0
$$

which holds for all $\tau_{M} \in[0,1]$. Finally, the requirements (12) from Assumption 2 hold if

$$
\tau_{M}<\min \left\{\frac{1}{2 \sqrt{2(0.6469)(0.5)}}, \frac{1}{4(0.5)}\right\}=0.5
$$

so all of our assumptions hold if $\tau_{M}<0.5$.

To illustrate our findings, we ran Mathematica simulations with $\tau_{M}=0.5$ and $\tau_{f}=0$, which produces the closed loop dynamics

$$
\left\{\begin{array}{l}
\dot{s}(t)=\frac{0.125}{1+(s(0.5\lfloor 2 t\rfloor))^{2}}[1-s(t)]-\frac{0.5 s(t)}{1+s^{2}(t)} x(t) \\
\dot{x}(t)=\left[\frac{0.5 s(t)}{1+s^{2}(t)}-\frac{0.125}{1+(s(0.5\lfloor 2 t\rfloor))^{2}}\right] x(t) .
\end{array}\right.
$$

where

$$
\lfloor a\rfloor=\max \{j \in\{0,1,2, \ldots\}: j \leq a\}
$$

is the floor function. In Figures 1-3 below, we plot the components of the state and the control values for three different initial conditions. The control values $D(t)$ appear as jagged lines in the plots because of the 0.5 sampling time in the control. In each case, the state vector converges to $\left(s_{*}, s_{\text {in }}-s_{*}\right)=(0.25,0.75)$, so our simulations help validate our theoretical findings.

\section{CONCLUSIONS}

We provided a new feedback analysis for two state chemostats whose uptake functions are not necessarily monotone, including cases where the uptake functions are of

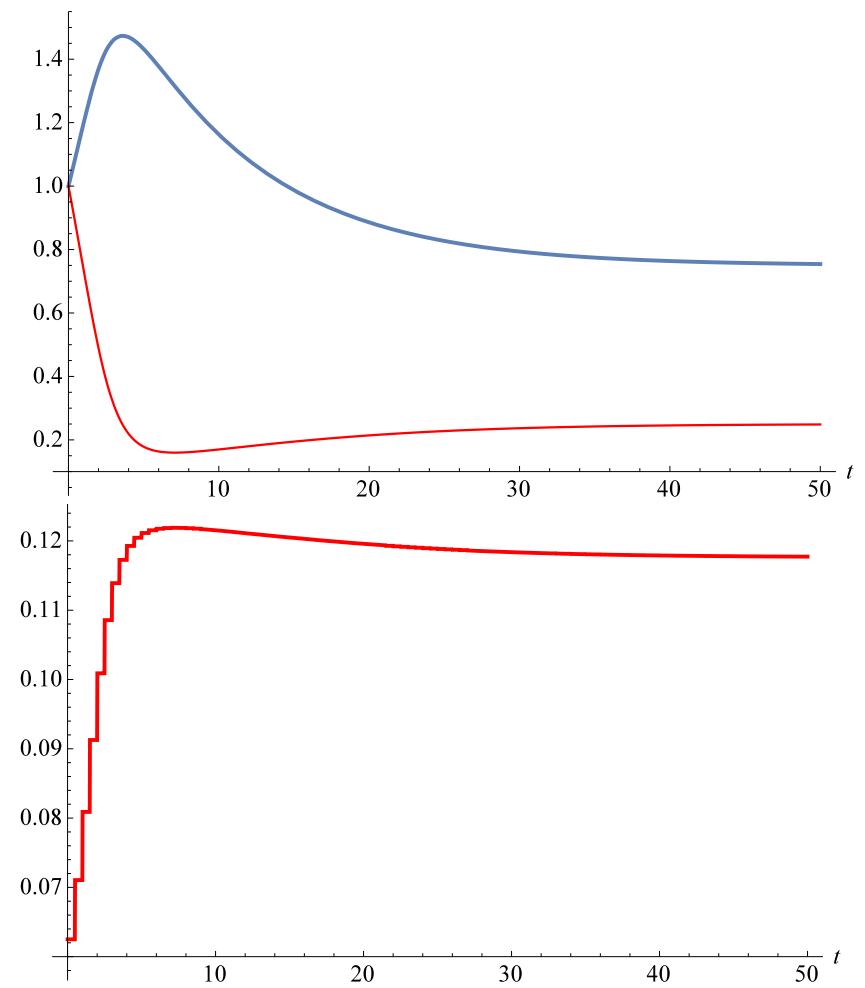

Fig. 1. Top: Solution $(s(t), x(t))$ of $(51)$ for Initial State $(1,1)$ Converging to $(0.25,0.75)$ with $\tau_{M}=0.5$ with Substrate $s(t)$ in Red and Species $x(t)$ in Blue. Bottom: Control Values $D(t)$.

Haldane type. Our new feedback only requires piecewise constant delayed measurements of the substrate level, and it ensures global asymptotic stability to an equilibrium point. Our convergence proof was based on a novel LyapunovKrasovskii functional method. In future work, we hope to exploit our Lyapunov-Kraovskii functional decay conditions to generalize our analysis to chemostats with several competing species with uncertainties [6] acting on the system.

\section{APPENDIX: PROOF OF LEMMA 1}

We construct functions $\mu_{1}$ and $\gamma$ that satisfy the requirements of Lemma 1 . Let $\varepsilon \in\left(0, s_{M} / 2\right)$ be a constant such that $\varepsilon \max \left\{\mu^{\prime}(\ell): 0 \leq \ell \leq s_{M}\right\}<2 \mu\left(s_{M} / 2\right)$ and set

$$
\begin{aligned}
\mu_{1}(s)= & \left(\max \left\{0, s-s_{M}+\varepsilon\right\}\right)^{2} \\
& + \begin{cases}\mu(s), & 0 \leq s \leq s_{M} \\
2 \mu\left(s_{M}\right)-\mu(s), & s>s_{M}\end{cases}
\end{aligned}
$$

and

$$
\gamma(s)=\frac{\mu_{1}(\max \{0, s\})}{\mu(\max \{0, s\})}-1
$$

for all $s>0$ and $\gamma(s)=0$ for all $s \leq 0$. If $s \leq s_{M}-\varepsilon$, then $\gamma(s)=0$. If $s \in\left(s_{M}-\varepsilon, s_{M}\right]$, then

$$
\begin{aligned}
\gamma^{\prime}(s)= & \frac{1}{\mu^{2}(s)}\left[\mu(s)\left(2\left(s-s_{M}+\varepsilon\right)+\mu^{\prime}(s)\right)\right. \\
& \left.-\left(\left(s-s_{M}+\varepsilon\right)^{2}+\mu(s)\right) \mu^{\prime}(s)\right] \\
\geq & \frac{1}{\mu^{2}(s)}\left(s-s_{M}+\varepsilon\right)[2 \mu(s) \\
& \left.-\left(s-s_{M}+\varepsilon\right) \mu^{\prime}(s)\right] \\
\geq & \frac{1}{\mu^{2}(s)}\left(s-s_{M}+\varepsilon\right)\left[2 \mu\left(s_{M} / 2\right)\right. \\
& \left.-\varepsilon \max \left\{\mu^{\prime}(\ell): 0 \leq \ell \leq s_{M}\right\}\right]>0 .
\end{aligned}
$$



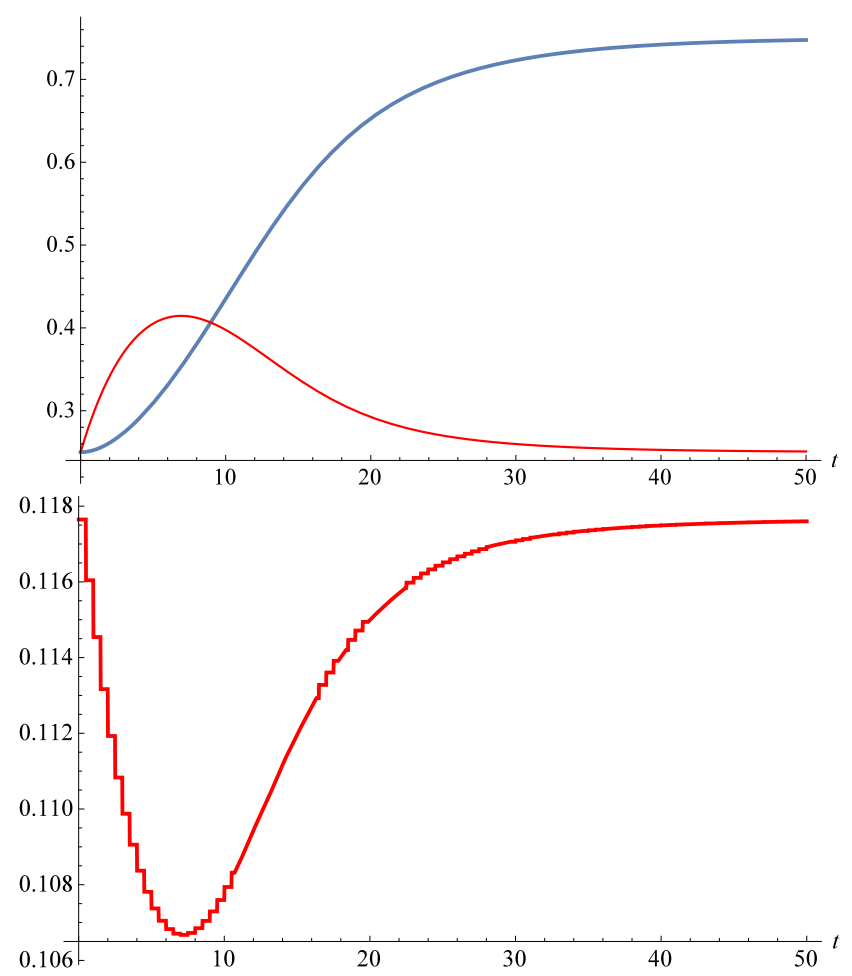

Fig. 2. Top: Solution $(s(t), x(t))$ of $(51)$ for Initial State $(0.25,0.25)$ Converging to $(0.25,0.75)$ with $\tau_{M}=0.5$ with Substrate $s(t)$ in Red and Species $x(t)$ in Blue. Bottom: Control Values $D(t)$.

If $s>s_{M}$, then $\mu^{\prime}(s) \leq 0$, so

$$
\begin{aligned}
\gamma^{\prime}(s)= & \frac{1}{\mu^{2}(s)}\left[\mu(s)\left[2\left(s-s_{M}+\varepsilon\right)-\mu^{\prime}(s)\right]\right. \\
& \left.-\left[2 \mu\left(s_{M}\right)-\mu(s)+\left(s-s_{M}+\varepsilon\right)^{2}\right] \mu^{\prime}(s)\right] \\
> & 0,
\end{aligned}
$$

since $\mu\left(s_{M}\right)>0$. Hence, $\gamma$ is nondecreasing, and $\mu_{1}$ and $\gamma$ satisfy our requirements.

\section{REFERENCES}

[1] G. Butler, S. Hsu, and P. Waltman. A mathematical model of the chemostat with periodic washout rate. SIAM Journal on Applied Mathematics 45(3):435-449, 1985. DOI:10.1137/0145025.

[2] A. Drame, M. Kothari and P. Wolenski. Almost periodic solutions of distributed parameter biochemical systems with time delay and time varying input. Electronic Journal of Differential Equations (2013)161:1-11, 2013

[3] E. Fridman, A. Seuret and J-P. Richard. Robust sampled-data stabilization of linear systems: an input delay approach. Automatica 40(8):1441-1446, 2004. DOI: 10.1016/j.automatica.2004.03.003.

[4] C. Fritsch, J. Harmand, and F. Campillo. A modeling approach of the chemostat. Ecological Modelling 299:1-13, 2015. DOI: 10.1016/j.ecolmodel.2014.11.021.

[5] J.-L. Gouzé and G. Robledo. Feedback control for nonmonotone competition models in the chemostat. Nonlinear Analysis: Real World Applications 6(4):671-690, 2005. DOI: 10.1016/j.nonrwa.2004.12.003.

[6] J.-L. Gouze and G. Robledo. Robust control for an uncertain chemostat model. Internat. Journal of Robust and Nonlinear Control 16(3):133155, 2006. DOI: 10.1002/rnc.1047.

[7] V. Lemesle and J.-L. Gouze. A simple unforced oscillatory growth model in the chemostat. Bulletin of Mathematical Biology 70(2):344357, 2008. DOI: 10.1007/s11538-007-9254-5.

[8] F. Mazenc, J. Harmand, and H. Mounier. Global stabilization of the chemostat with delayed and sampled measurements and control. In Proceedings of the 9th IFAC Symposium on Nonlinear Control Systems, Toulouse, France, pp. 170-174, 2013. DOI: 10.3182/201309043-FR-2041.00049.

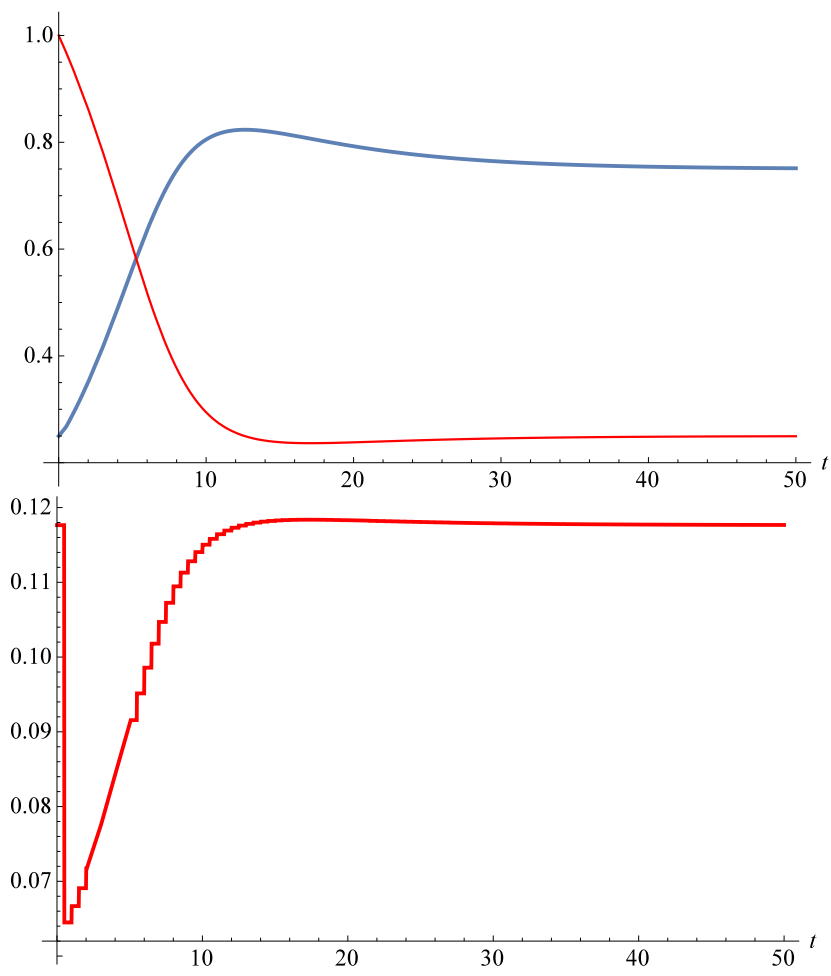

Fig. 3. Top: Solution $(s(t), x(t))$ of (51) for Initial State $(1,0.25)$ Converging to $(0.25,0.75)$ with $\tau_{M}=0.5$ with Substrate $s(t)$ in Red and Species $x(t)$ in Blue. Bottom: Control Values $D(t)$.

[9] F. Mazenc and M. Malisoff. Stabilization of a chemostat model with Haldane growth functions and a delay in the measurement. Automatica 46(9):1428-1436, 2010. DOI: 10.1016/j.automatica.2010.06.012.

[10] F. Mazenc, M. Malisoff, and T. Dinh. Robustness of nonlinear systems with respect to delay and sampling of the controls. Automatica 49(6):1925-1931, 2013. DOI: 10.1016/j.automatica.2013.02.064.

[11] J. Monod. La technique de culture continue théorie et applications. Annales de l'Institut Pasteur 79:390-410, 1950. DOI: 10.1016/B9780-12-460482-7.50023-3.

[12] A. Novick and L. Szilard. Description of the chemostat. Science 112(2920):715-716, 1950. DOI: 10.1126/science.112.2920.715/

[13] S. Pilyugin and P. Waltman. Competition in the unstirred chemostat with periodic input and washout. SIAM Journal on Applied Mathematics 59(4):1157-1177, 1999. DOI: 10.1137/S0036139997323954.

[14] G. Robledo. Feedback stabilization for a chemostat with delayed output. Mathematical Biosciences and Engineering 6(3):629-647, 2009. DOI: $10.3934 / \mathrm{mbe} .2009 .6 .629$.

[15] G. Robledo, F. Grognard, J.-L. Gouze. Global stability for a model of competition in the chemostat with microbial inputs. Nonlinear Analysis: Real World Applications 13(2):582-598. DOI: 10.1016/j.nonrwa.2011.07.049.

[16] H. Smith and P. Waltman. The Theory of the Chemostat. Cambridge University Press, Cambridge, UK, 1995.

[17] H. Xia, G. Wolkowicz, and L. Wang. Transient osciallations induced by delayed growth response in the chemostat. Journal of Mathematical Biology 50(5):489-530, 2005. DOI: 10.1007/s00285-004-0311-5. 